\title{
A numerical scheme for recovering the nonlinear characteristics of a single degree of freedom structure: non-parametric system identification
}

\author{
J. Park ${ }^{1}$, T. S. Jang ${ }^{1}$, S. Syngellakis ${ }^{2}$ \& H. G. Sung ${ }^{3}$ \\ ${ }^{I}$ Department of Naval Architecture and Ocean Engineering, \\ Pusan National University, Republic of Korea \\ ${ }^{2}$ Wessex Institute of Technology, UK \\ ${ }^{3}$ Maritime and Ocean Engineering Research Institute (MOERI; formerly \\ KRISO) of KIOST (formerly KORDI), Republic of Korea
}

\begin{abstract}
The aim of this paper is to present a numerical scheme for the identification of the nonlinear characteristics of a dynamically excited, single degree of freedom structure, using a non-parametric procedure, recently proposed by the second author; this involves the simultaneous identification of the nonlinear characteristics of both damping and restoring force in dynamic systems whose damping depends on velocity alone. According to this method, the response of the structure is first measured then an integral equation accounting for its unknown nonlinear characteristics is derived. This is an integral equation of the first kind, involving numerical instability in the Hadamard sense. To overcome this difficulty, the Landweber regularization, combined with the L-curve criterion, is applied to the integral equation. Adopting a dynamic model for a test structure, the corresponding nonlinear system identification is achieved through the proposed numerical solution of the governing integral equation.

Keywords: non-parametric system identification, nonlinear damping, nonlinear stiffness, dynamic response data, single-degree-of-freedom.
\end{abstract}




\section{Introduction}

In the last two decades, there has been a lot of research work on the subject of dynamic system identification conducted by Masri et al. [1], Chassiakos and Masri [2], Liang et al. [3, 4], Spina et al. [5], Iourtchenko and Dimentberg [6], and others [7-17]. However, most of this research has identified the nonlinear characteristics of either damping or the restoring force. Only in a few recent studies, there has been an attempt to identify the nonlinear characteristics of both $[18,19]$.

In this paper, the nonlinear characteristics of both damping and restoring force of a single degree of freedom system are determined. For this purpose, a non-parametric procedure according to Jang's method [20] is applied; this involves a non-parametric, simultaneous identification of the nonlinear characteristics of both damping and restoring force for systems whose damping depends on velocity alone. The nonlinear damping and restoring force parameters of a system with time dependent damping are numerically obtained. A sinusoidal (with respect to velocity) damping as well as a sinusoidal nonlinear restoring force model is adopted for such a system.

\section{Motion responses}

A nonlinear single degree of freedom system is here considered; its damping is assumed to depend on velocity alone. Although a physical experiment should be conducted to provide measurements of the system response for the system identification process, virtual measurements of the velocity and the displacement are here numerically obtained using the Runge-Kutta method.

The system is governed by the nonlinear ordinary differential equation

$$
m \ddot{y}+k y=f(\dot{y})+r(y)
$$

with the following initial conditions [20],

$$
y(0)=\alpha, \quad \dot{y}(0)=\beta,
$$

where the constants $m$ and $k$ denote the mass of a particle of the oscillator and a spring coefficient, respectively. The velocity dependent nonlinear damping $f(\dot{y})$ and the nonlinear restoring force $r(y)$ are assumed anti-symmetric:

$$
\begin{gathered}
f(-\dot{y})=-f(\dot{y}), \\
r(-y)=-r(y) .
\end{gathered}
$$

The term $f(\dot{y})$ in eqn (1) indicates a general form of velocity-dependent nonlinear damping for conventional dampers; this is the usual damping modelling of nonlinear systems that is adopted in natural sciences. In the engineering field, when ships or other floating bodies are subjected to large roll motions in waves, either when sailing or when at anchor, they experience nonlinear roll damping, which can be characterized as a type of velocitydependent damping of the form $f(\dot{y})$ in eqn (1). This phenomenon is usually caused physically by the effects of nonlinear free-surface wave making and the 
nonlinear viscous dissipation of fluid flow. In contrast to this type of damping, the nonlinear damping characterized by $f(y) \dot{y}$ in the Liénard equation appeared in the early research investigation carried out by Jang et al. [18]; simultaneous nonlinear identification was carried out there for the Van der Pol nonlinear equation. The other term that appears in eqn (1), namely $r(y)$, represents a general form of the nonlinear restoring force of the oscillator.

In this paper, the mixed linear-cubic (with respect to $\dot{y}$ ) damping model is applied and the linear-cubic nonlinear restoring force model as follows:

$$
f(y)=a \sin (\dot{y}) \text { and } r(y)=c \sin (y),
$$

where the adopted values for the coefficients are $a=-3.385$ and $c=-20.3$ (table 1). The assumed initial conditions in eqn (2) are

$$
y(0)=1.0, \dot{y}(0)=0 \text {. }
$$

Table 1: Principal properties of the numerical experiment.

\begin{tabular}{llc}
\hline \multicolumn{3}{c}{ Principal properties [25] } \\
\hline Governing equation & $m \ddot{y}+k y=a \sin (\dot{y})+c \sin (y)$ \\
Mass (kg) & $m$ & 6.407 \\
Linear restoring coefficient (N/m) & $k$ & 20 \\
Nonlinear damping coefficient (Ns/m) & $a$ & -3.385 \\
Nonlinear restoring coefficient $(\mathrm{N} / \mathrm{m})$ & $c$ & -20.3 \\
\hline
\end{tabular}

For the numerical calculation of the system response, the Runge-Kutta method is used for the solution of eqn (1) and the trapezoidal rule is applied for the numerical integration; the time domain is taken as $0 \leq t \leq 20 \mathrm{~s}$ and the total number of time intervals is 401 or $\Delta t=0.05 \mathrm{~s}$.

Fig. 1 shows the displacement and velocity obtained by solving eqn (1) for the damping and restoring force as well as the initial conditions given by eqns. (5) and (6), respectively; fig. 2 depicts the phase diagram corresponding to the solution of fig. 1 .

Now, the original nonlinear differential equation (1) is transformed to an equivalent nonlinear Volterra integral equation as follows:

$$
y(t)=\frac{\alpha}{\mu} y_{1}(t)+\frac{\beta}{v} y_{2}(t)+\int_{0}^{t} \frac{y_{1}(\tau) y_{2}(t)-y_{1}(t) y_{2}(\tau)}{m W(\tau)}[f(\dot{y})+r(y)] \mathrm{d} \tau,
$$

in which $y_{1}$ and $y_{2}$ are chosen so that they satisfy

$$
\begin{array}{ll}
m \ddot{y}_{1}+k y_{1}=0, & y_{1}(0)=\mu, \dot{y}_{1}(0)=0, \\
m \ddot{y}_{2}+k y_{2}=0, & y_{2}(0)=0, \dot{y}_{2}(0)=v,
\end{array}
$$

and

$$
W(\tau)=y_{1}(\tau) \dot{y}_{2}(\tau)-\dot{y}_{1}(\tau) y_{2}(\tau)
$$

The values of $\mu$ and $v$ are assumed to have the following magnitude in this paper $[7,18,20]: \mu=v=1$. 

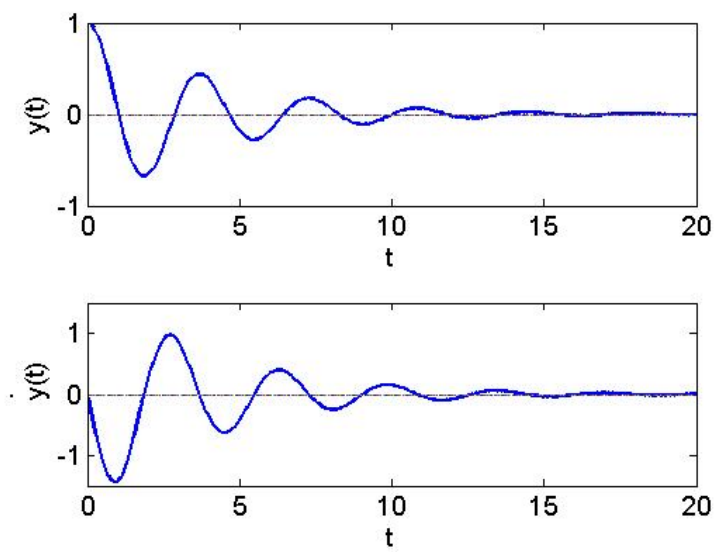

Figure 1: System responses obtained by solving eqns (1), (5) and (6).

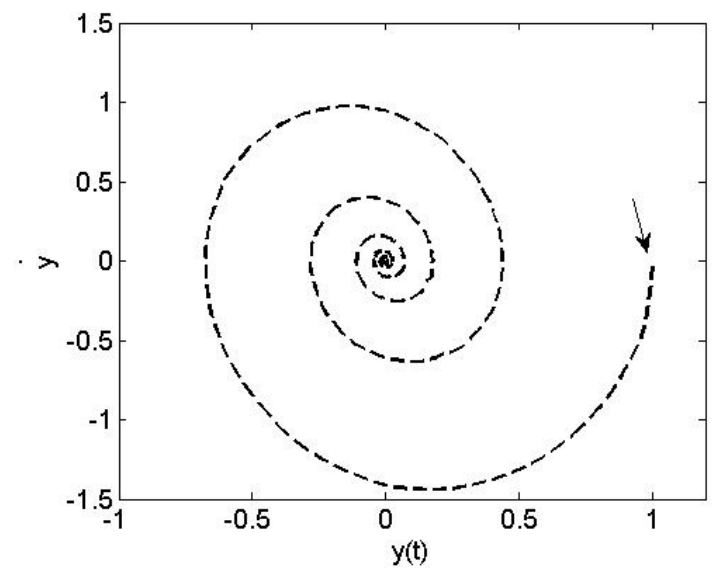

Figure 2: System phase diagram associated with the solution of fig. 1

It is then possible to construct an integral equation for $u$, given by

$$
u=f(\dot{y})+r(y) \text {, }
$$

using eqn (7):

$$
y(t)-\alpha y_{1}(t)-\beta y_{2}(t)=\int_{0}^{t} K(t, \tau) u(\tau) \mathrm{d} \tau .
$$

with the kernel $K$ defined as

$$
K(t, \tau)=\frac{y_{1}(\tau) y_{2}(t)-y_{1}(t) y_{2}(\tau)}{m W(\tau)} .
$$


The relationship in eqn (10) is classified as a Volterra-type integral equation of the first kind [20], which defines an inverse problem for the simultaneous identification of the velocity-dependent nonlinear damping and restoring force together with the nonlinear function defined by eqn (9). Eqn (10) and eqn (9) constitute the required equations of the present nonlinear inverse problem for the two unknown functions, $f$ and $r$.

\section{Calculating $\boldsymbol{u}$ by regularization}

In the previous section, the analysis was based on the assumption that $u$ in eqn (9) is known. As far as the determination of $u$ is concerned, an integral equation of the first kind, such as eqn (10), must be dealt with. Thus, the question of whether the solution to system identification depends on the measured response data in a continuous manner remains. This question can be answered by the theory of integral equations, according to which, if integral equations with a regular kernel such as the $K$ given by eqn (11) are of the first kind, then their solutions lack stability properties [21-23]. Unreliable solutions may result from a small amount of noisy data due to the considerable amplification of a small noisy error.

To overcome the difficulty of the solution's instability with regard to $f$ and $r$, the Landweber regularization

$$
u_{j}=u_{j-1}-\lambda L^{*}\left\{L\left(u_{j-1}\right)\right\}+\lambda L^{*}(\eta), j=1,2 \ldots .
$$

is applied to prevent it from affecting the performance of the identification [18, $20,23]$. In eqn (12), $L$ is the operator,

$$
L(z)=\int_{0}^{t} K(t, \tau) z(\tau) d \tau,
$$

$L^{*}$ denotes the adjoint operator of $L, \lambda$ is a positive constant and $\eta$ is given by

$$
\eta=y-\alpha y_{1}-\beta y_{2}
$$

In the theory of iterative regularization, an appropriate number of iterations $j$ is important for an accurate solution. Here, the L-curve criterion is applied to find an appropriate number of iterations. [18, 20, 22, 24].

\section{Simultaneous identification}

This section contains an illustration of the way by which the two unknowns, that is, the velocity-dependent nonlinear damping $f(\dot{y})$ and nonlinear restoring force $r(y)$, can be identified using the formulated eqns (9) and (10).

It is initially assumed that the measured response data for $y$ and $\dot{y}$ of the nonlinear oscillation governed by eqn (1) are depicted as shown in fig. 1; a number of the intersection points of the curve of $y$ with the $t$-axis are found at times $t=t_{1}, t_{2}, \ldots$; the $t_{i}$ for $i=1,2, \ldots$ are zero-crossing times for the displacement. In exactly the same way, there are also intersection points of the curve of $\dot{y}$ with the $t$-axis at $t=t^{1}, t^{2}, \ldots$, which are zero-crossing times for the velocity; hence, 


$$
\begin{array}{ll}
y\left(t_{i}\right)=0 & \text { for } i=1,2, \ldots \\
\dot{y}\left(t^{k}\right)=0 & \text { for } k=1,2, \ldots
\end{array}
$$

Since eqn (9) remains valid for any positive time, it should also hold not only for zero-crossing times (for the displacement) $t=t_{1}, t_{2}, \ldots$, but also for zero-crossing times (for the velocity) $t=t^{1}, t^{2}, \ldots$ Thus, it follows that

$$
u\left(t_{i}\right)=f\left[\dot{y}\left(t_{i}\right)\right]+r\left[y\left(t_{i}\right)\right],
$$

and

$$
u\left(t^{k}\right)=f\left[\dot{y}\left(t^{k}\right)\right]+r\left[y\left(t^{k}\right)\right] .
$$

From the information of the measured response data for $y$ and $\dot{y}$, the zerocrossing times of the displacement $t_{i}(i=1,2, \ldots)$ and the velocity $t^{k}(k=1,2, \ldots)$ and the stabilized $u$ in eqn (10), it follows that the nonlinear damping at times $t_{i}$ is

$$
u\left(t_{i}\right)=f\left[\dot{y}\left(t_{i}\right)\right]
$$

and the nonlinear restoring force at $t^{k}$ is

$$
u\left(t^{k}\right)=r\left[y\left(t^{k}\right)\right] \text {. }
$$

Thus, the decoupled eqns (19) and (20) for the two unknowns, $f$ and $r$, respectively, have been reached.

\section{Numerical results}

The principal properties of the numerical experiment are shown in table 1 . In this virtual experiment, the measurement noise level of the motion response is assumed to be $\delta=0.01$. As already mentioned, the Runge-Kutta method is applied for the determination of the system response shown in fig. 1. The optimal or appropriate iteration number $j_{\text {opt }}=4 \times 10^{5}$ of the Landweber's regularization method, corresponding to the corner of the L-curve, and the respective $u_{j, \text { opt }}$, determined by solving eqn (10) with noise $\delta=0.01$ are depicted in fig. 3 and fig. 4, respectively.

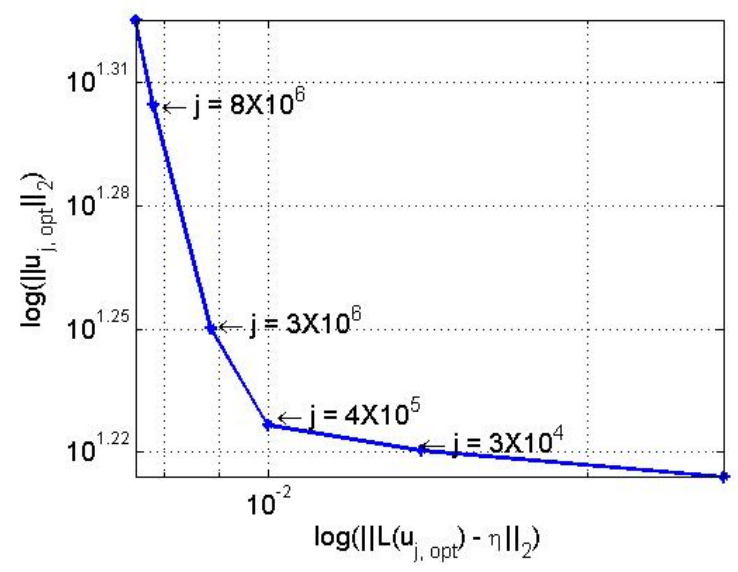

Figure 3: L-curve for the records of fig 1: optimal iteration number $j_{\text {opt }}=4 \times 10^{5}$. 


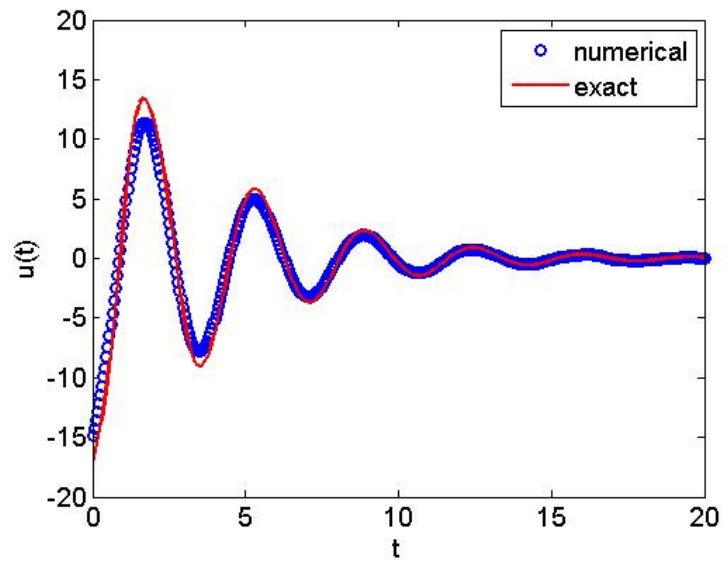

Figure 4: Computed $u_{j, \text { opt }}(t)$ for the optimal iteration number $j_{\text {opt }}=4 \times 10^{5}$.

Employing the $u_{j, \text { opt }}$ and the zero-crossing times for the displacement $t_{i}$ and velocity $t^{k}$ (fig. 5), the determined nonlinear damping $f(\dot{y})$ and the nonlinear restoring force $r(y)$ are demonstrated in fig. 6 and fig. 7, respectively.
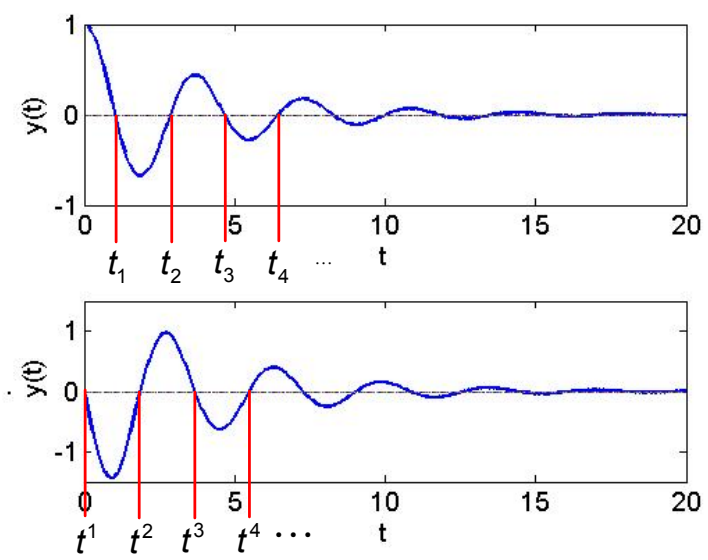

Figure 5: System responses with the zero-crossing times.

\section{Conclusions}

In the present study, Jang's method is applied for the numerical identification of the nonlinear damping and the nonlinear restoring forces in a SDOF dynamic structure. The main idea of the Jang's method relies on zero-crossing time measurements from experimental displacement and velocity records and it is a 
very simple but accurate method for the identification of nonlinear characteristics. Of course, it has an ill-posedness property which causes solution instability; but this can be handled by a regularization method such as the Landweber's method with the L-curve criterion. Finally, the suitability of Jang's method for the determination of the nonlinear characteristics of a SDOF dynamic structure has been demonstrated through a numerical experiment.

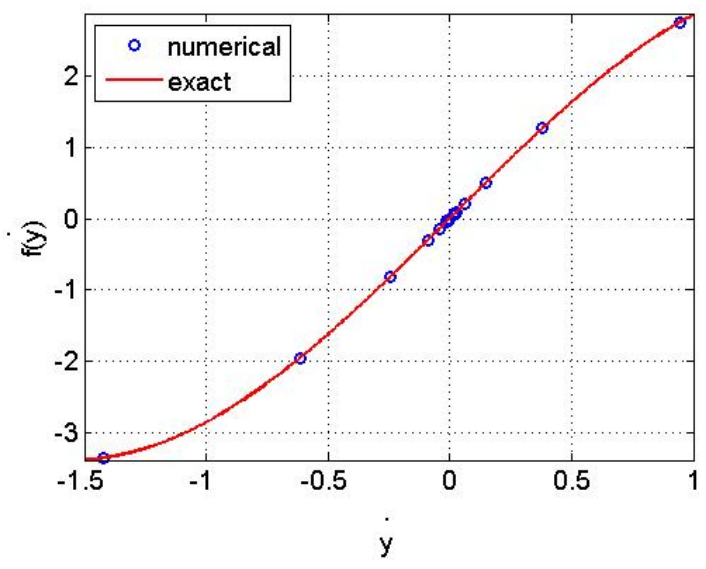

Figure 6: Numerical results for $f(\dot{y})$ compared to the originally assumed nonlinear damping $f(\dot{y})=a \sin (\dot{y}) ; a=-3.385$.

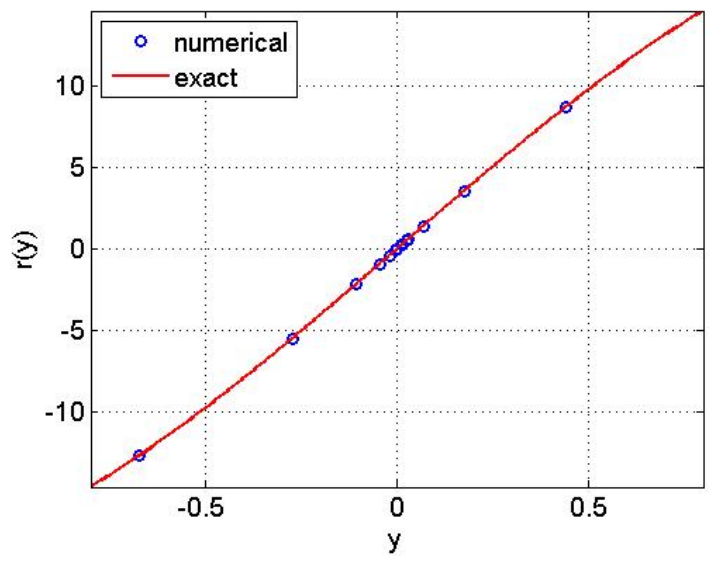

Figure 7: Numerical results for $r(y)$ compared to the originally assumed nonlinear restoring force $r(y)=c \sin (y) ; c=-20.3$. 


\section{Acknowledgements}

The first and the second authors are supported by Basic Science Research Program through the National Research Foundation of Korea (NRF) funded by the Ministry of Education (Grant No. 2011-0010090). The fourth author acknowledges the financial support by a principal R\&D program of KIOST: "Performance Evaluation Technologies of Offshore Operability for Transport and Installation of Offshore Structures".

\section{References}

[1] Masri, S.F., Chassiakos, A.G. \& Cauchey, T.K., Identification of nonlinear dynamic systems using neural networks. Journal of Applied Mechanics, 60, pp. 123-133, 1993.

[2] Chassiakos, A.G. \& Masri, S.F., Modeling unknown structural systems through the use of neural networks. Earthquake Engineering and Structural Dynamics, 25, pp. 117-128, 1996.

[3] Liang, Y.C., Zhou, C.G. \& Wang, Z.S., Identification of restoring forces in non-linear vibration systems based on neural networks. Journal of Sound and Vibration, 206, pp. 103-108, 1997.

[4] Liang, Y.C., Feng, D.P. \& Cooper, J.E., Identification of restoring forces in non-linear vibration systems using fuzzy adaptive neural networks. Journal of Sound and Vibration, 242, pp. 47-58, 2001.

[5] Spina, D., Valente, C. \& Tomlinson, G.R., A new procedure for detecting nonlinearity from transient data using the Gabor transform. Nonlinear Dynamics, 11, pp. 235-254, 1996.

[6] Iourtchenko, D.V. \& Dimentberg, M.F., In-service identification of nonlinear damping from measured random vibration. Journal of Sound and Vibration, 255, pp. 549-554, 2002.

[7] Feldman, M., Considering high harmonics for identification of non-linear systems by Hilbert transform. Mechanical Systems and Signal Processing, 2 pp. 943-958, 2007.

[8] Worden, K., Hickey, D., Haroon, M. \& Adams, D.E., Nonlinear system identification of automotive dampers: A time and frequency-domain analysis. Mechanical Systems and Signal Processing, 23, pp. 104-126, 2009.

[9] Maiaa, N.M.M. \& Ewins, D.J, A new approach for the modal identification of lightly damped structures. Mechanical Systems and Signal Processing, 3, pp. 173-193, 1989.

[10] Iourtchenko, D.V., Duval, L. \& Dimentberg, M.F., The damping identification for certain SDOF systems. Developments in Theoretical and Applied Mechanics, Proceedings of SECT AM XX Conference, Auburn University, 2000.

[11] Jang, T.S., Kwon, S.H. \& Han, S.L., A novel method for non-parametric identification of nonlinear restoring forces in nonlinear vibrations from 
noisy response data: a conservative system. Journal of Mechanical Science and Technology, 23(11), pp. 2938-2947, 2009.

[12] Jang, T.S., Son, J.W., Han, S.L., Sung, H.J., Lee, S.K. \& Shin, S.C., A numerical investigation on nonparametric identification of nonlinear roll damping moment of a ship from transient response. The Open Ocean Engineering Journal, 3 pp. 100-107, 2010.

[13] Jang, T.S., A novel method for the non-parametric identification of nonlinear restoring forces in nonlinear vibrations based on response data: a dissipative nonlinear dynamical system. Ships and Offshore Structures, 6(4), pp. 257-263, 2011.

[14] Jang, T.S. Baek, H.S. Kim, M.C. Moon, B.Y. A new method for detecting the time-varying nonlinear damping in nonlinear oscillation systems: nonparametric identification. Mathematical Problems in Engineering, 2011, DOI:10.1155/2011/749309, 2011.

[15] Jang, T.S., A new simultaneous identification of the harmonic excitations and nonlinear damping of forced damped nonlinear oscillations: a parametric approach. Journal of Applied Mathematics, 2013, DOI:10.1155/2013/754576, 2013.

[16] Jang, T.S., A method for simultaneous identification of the full nonlinear damping and the phase shift and amplitude of the external harmonic excitation in a forced nonlinear oscillator. Computers \& Structures, 120(15), pp. 77-85, 2013.

[17] Jang, T.S., Uniqueness and stability of the simultaneous detection of the nonlinear restoring and excitation of a forced nonlinear oscillation. Applied Mathematics and Computation, 228, pp. 234-239, 2014.

[18] Jang, T.S., Choi, H.S. \& Han, S.L., A new method for detecting non-linear damping and restoring forces in non-linear oscillation systems from transient data. International Journal of Non-Linear Mechanics, 44, pp. 801-808, 2009.

[19] Jang, T.S., Kwon, S.H. \& Lee, J.H., Recovering the functional form of the nonlinear roll damping of ships from a free-roll decay experiment: an inverse formulism. Ocean Engineering, 37(14-15), pp. 1337-1344, 2010.

[20] Jang, T.S., Non-parametric simultaneous identification of both the nonlinear damping and restoring characteristics of nonlinear systems whose dampings depend on velocity alone. Mechanical Systems and Signal Processing, 25(4), pp. 1159-1173, 2011.

[21] Tikhonov, A.N., Solution of incorrectly formulated problems and the regularization method. Soviet Math. Dokl., 4, pp. 1035-1038, 1963.

[22] Groetsch, C.W., Inverse Problems in the Mathematical Sciences, Vieweg, 1993.

[23] Landweber, L., An iteration formula for Fredholm integral equations of the first kind. American Journal of Mathematics, 73, pp. 615-624, 1951.

[24] Kirsch, A., An Introduction to the Mathematical Theory of Inverse Problems, Springer, 1996.

[25] Lee, S.K., An introduction to towing Tank Experiment: Ship Motion and Manoeuvrability, Pusan National University press, 2008. 